\title{
High Energy and Spatial Resolution EELS Band Gap Measurements Using a Nion Monochromated Cold Field Emission HERMES Dedicated STEM
}

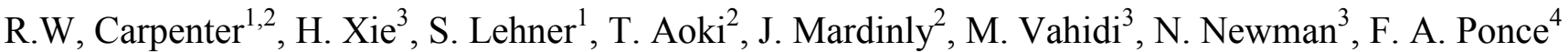 \\ ${ }^{1}$ Dept. of Chemistry and Biochemistry, Arizona State University, Tempe, USA \\ ${ }^{2}$ LeRoy Eyring Center for Solid State Science, Arizona State University, Tempe, USA \\ ${ }^{3}$ School for Engr. of Matter, Transport, and Energy, Arizona State University, Tempe, USA \\ ${ }^{4}$ Dept. of Physics, Arizona State University, Tempe, USA
}

Local measurement of band gaps in nanomaterials is an intriguing experimental possibility, but until recently not possible because both high spatial resolution and high EELS energy resolution with small zero loss peak (ZLP) tails are required. The addition of a monochromator to a Nion probe corrected, cold FEG source D-STEM microscope (denoted HERMES) fitted with a Gatan Enfinium spectrometer has produced the EELS performance required for band gap measurements down less than $1 \mathrm{eV}$. The microscope, and its ultrastable installation site have been described [1,2]. ZLPs (normalized to the same intensity) from this microscope (in red) and another probe corrected Schottky FEG source but nonmonochromated AEM located in our laboratory are compared in Figure 1 and the data are summarized in Table 1. The $37 \mathrm{meV}$ energy resolution of the monochromated cold FEG source Nion is significantly better than the typical Schottky FEG $950 \mathrm{meV}$ resolution as shown in Table 1 and the energy tails at lower intensity are reduced. The resolution and beam tails depend somewhat on microscope alignment and collection time. The Nion ZLP in Figure 1 is the sum of 15 one second spectra. Shorter collection times ( 0.1 second, 1 spectrum) result in higher energy resolution (14 meV at FWHM) and smaller extended tails ( $54 \mathrm{meV} \mathrm{FW}$ at 1\% maximum intensity). Kimoto et al have shown that both CFEG and a monochromated Schottky source are useful for measuring band gaps, but both of these have more extended low loss tails than the HERMES monochromated CFEG, particularly the tunneling tail for the CFEG, which complicates small gap measurement [3]. Some first measurements are shown below.

The measurements of band gaps $\left(\mathrm{E}_{\mathrm{g}}\right)$ for an $\mathrm{Al}_{0.6} \mathrm{Ga}_{0.4} \mathrm{~N} / \mathrm{Al}_{0.75} \mathrm{Ga}_{0.25} \mathrm{~N}$ multiquantumwell structure deposited on an AIN substrate were 5.0 and $5.6 \mathrm{eV}$ for the MQW and the substrate, respectively. The MQW is shown is Fig 2a, and the easily visible difference in band gaps in Fig 2 b,c. The change between the two occurred over a distance of about $8 \mathrm{~nm}$, in reasonable agreement with Egerton's plasmon model for delocalization range [4]. The delocalization appears to occur mostly in the AIN substrate and thus unsymmetrical about the interface. The structure visible in the loss function is similar but not identical in the MQW and AIN; some can be interpreted as single electron excitation, but not all. Ion thinning was used for specimen prep, so there may be a damage component. Figure 3 shows the band gap $(5.7 \mathrm{eV})$ in hexagonal $\mathrm{BN}$ along with the $\pi(8.5 \mathrm{eV})$ and part of the $\pi+\sigma(25 \mathrm{eV})$ plasmons. The ZLP has been shifted partially off the detector to avoid many readouts, improving S/N. This shift is simple and useful for large band gaps, but difficult for small band gaps. In the latter case using higher dispersion is useful, but that requires higher system stability. Figure 4 shows the band gap for natural iron pyrite, $\mathrm{FeS}_{2}$. Pyrite is an interesting solar cell candidate film. In this case natural pyrite was used as a substrate to deposit synthetic pyrite single nanocrystals by a vapor deposition process. The band gap for the natural pyrite was $\sim 0.9 \mathrm{eV}$, and for the synthetic pyrite was from 0.7 to $0.8 \mathrm{eV}$. Since natural pyrite is typically impure and the synthetic product may have variable stoichiometry the correlation of band gap with composition is of special interest in this case. The small band gap of pyrite requires use of high dispersion with high stability to obtain best data. 


\section{References}

[1] R W Carpenter et al, Micros. Micoranal. 18(Supp 2) (2012), p. 408.

[2] O L Krivanek et al, Microscopy 62 (2013), p. 3.

[3] K Kimoto et al, Micron 36 (2005), p.185

[4] R F Egerton, Ultramicros. 107 (2007), p.575.

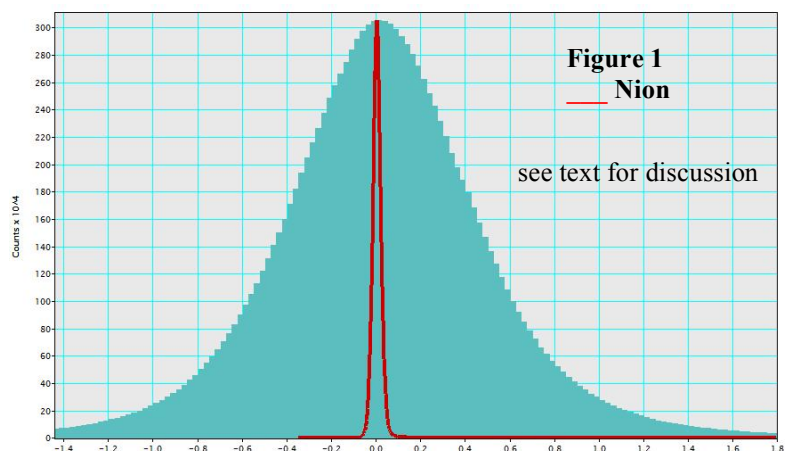

TABLE 1

Full width of zero loss peaks as function of zp intensity for the monochromated NION and an aberration corrected Schottky FEG STEM AEM. Both microscopes use the same type EELS spectrometer, a Gatan Enfinium.

\begin{tabular}{|l|c|c|c|c|}
\hline Per cent of Imax & 50 & 25 & 10 & 1 \\
\hline $\begin{array}{l}\text { NION, } \\
\text { FW @ } 0 \%, \mathrm{eV}\end{array}$ & 0.037 & 0.057 & 0.078 & 0.136 \\
\hline $\begin{array}{l}\text { AEM } \\
\text { FW@\% } \mathrm{eV}\end{array}$ & 0.95 & 1.40 & 1.98 & 3.90 \\
\hline
\end{tabular}

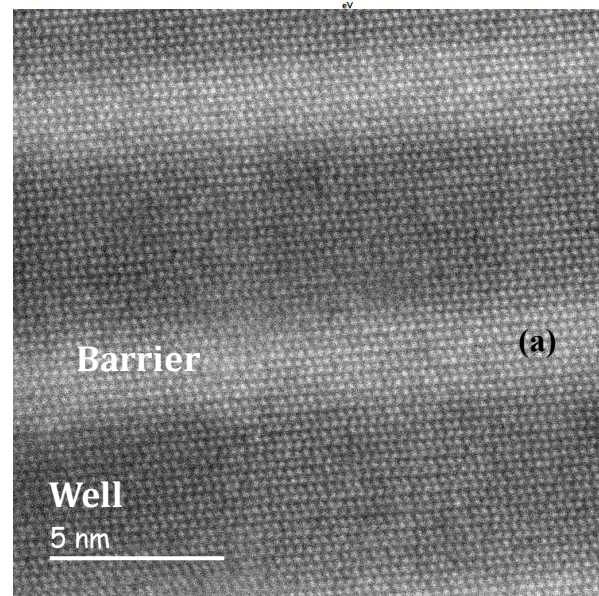

(b)

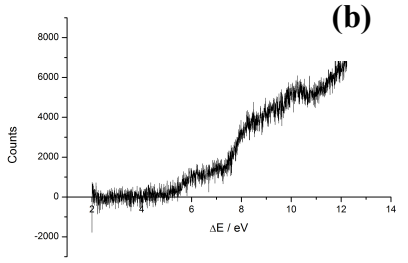

(c)

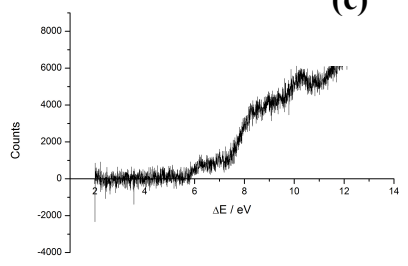

Figure 2. (a) AlGaN MQW structure, (b) MQW Eg $=5.0 \mathrm{eV}$, (c) AlN substrate Eg = $5.6 \mathrm{eV}$

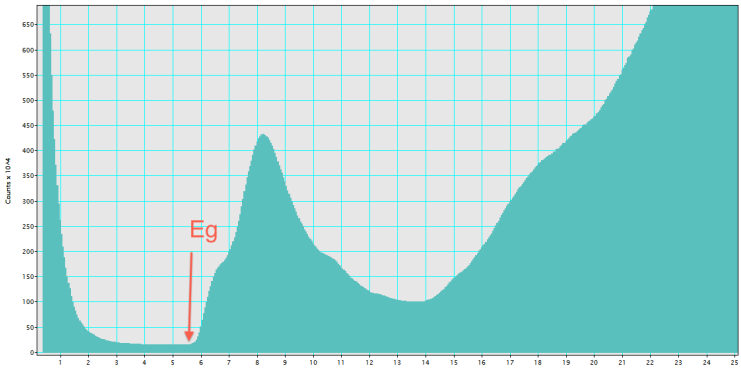

Figure $3 . \mathrm{E}_{\mathrm{g}}=5.7 \mathrm{eV}$ for $\mathrm{hBN}$

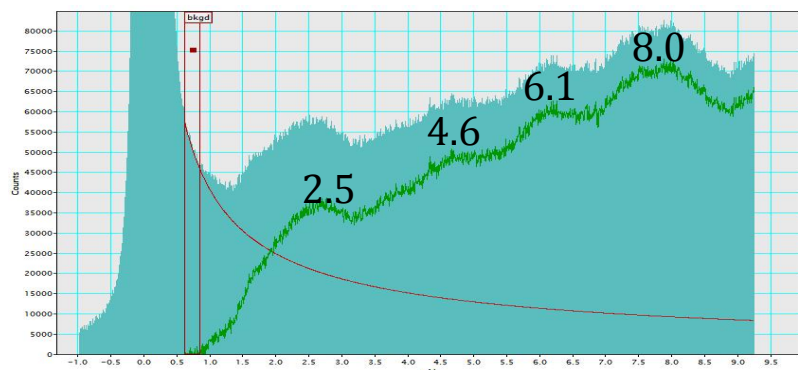

Figure 4. $\mathrm{E}_{\mathrm{g}} \approx 0.9 \mathrm{eV}$ for natural pyrite 